\title{
BMJ Open Perspectives of paediatric hospital staff on factors influencing the sustainability and spread of a safety quality improvement programme
}

\author{
Peter Lachman (D) , ${ }^{1}$ Dawid Gondek, ${ }^{2}$ Julian Edbrooke-Childs, ${ }^{2}$ Jessica Deighton, ${ }^{2}$ \\ Emily Stapley²
}

To cite: Lachman $P$, Gondek D, EdbrookeChilds J, et al. Perspectives of paediatric hospital staff on factors influencing the sustainability and spread of a safety quality improvement programme. BMJ Open 2021;11:e042163. doi:10.1136/ bmjopen-2020-042163

- Prepublication history for this paper is available online. To view these files, please visit the journal online (http://dx.doi. org/10.1136/bmjopen-2020042163).

Received 27 June 2020 Revised 20 February 2021 Accepted 04 March 2021
A) Check for updates

(C) Author(s) (or their employer(s)) 2021. Re-use permitted under CC BY-NC. No commercial re-use. See rights and permissions. Published by BMJ.

${ }^{1}$ Quality Improvement, Royal College of Physicians of Ireland, Dublin, Ireland

${ }^{2}$ Evidence Based Practice Unit, University College London and the Anna Freud National Centre for Children and families, London, UK, London, UK

Correspondence to

Dr Peter Lachman;

Peterlachman@rcpi.ie

\section{ABSTRACT}

Objective Situation Awareness For Everyone (SAFE) is a quality improvement programme aiming to improve situation awareness in paediatric clinical teams. The aim of our study was to examine hospital staff perceptions of the facilitators and barriers/challenges to the sustaining and subsequent spread of the huddle, the key intervention of the SAFE programme.

Setting Interviews were held on two wards in two children hospitals and on two children wards in two district general hospitals.

Method Semistructured interviews were conducted with 23 staff members from four National Health Service paediatric wards. A deductive thematic analysis was conducted, drawing on an existing framework, which groups the factors influencing programme sustainability into four categories: innovation, leadership, process and context.

Participants 23 staff in two children's hospitals and two children's wards across four UK hospitals, comprising of nurses and doctors, administration or housekeeping staff, ward managers and matrons, and allied professionals. Primary outcomes Understanding factors contributing to the sustaining and spread of a quality improvement intervention. Results Perceptions of the benefits, purpose and fit of the huddle, team commitment, sharing learning, adaptation of the method and senior leadership were identified as facilitators. High staff turnover, large multiple specialty medical staff teams, lack of senior leadership and dislike of change were identified as barriers/challenges.

Conclusions Sustaining and spreading quality improvement interventions in a complex clinical setting requires understanding of the interplay between the actual innovation and existing leadership, process and contextual factors. These must be considered at the planning stage of an innovation to maximise the potential for sustainability and spread to other settings.

\section{INTRODUCTION}

The Situation Awareness For Everyone (S.A.F.E.) programme has been introduced in 51 hospitals across England. Its aim is to improve situation awareness, including the introduction of patient safety theories and methods, through the routine use of 'the huddle' on paediatric wards. In this context,
Strengths and limitations of this study

- This study explores the factors that can facilitate the sustainability of improvement initiatives.

- This study adds to an existing framework for sustainability by also including factors that can improve the spread of improvement initiatives.

- The findings were derived from qualitative interviews with front-line staff (representing a range of professions) who participated in the improvement initiative at four hospitals.

- There are limitations in the transferability of the findings to other populations and settings, given the small sample of staff and hospitals included in this study.

- While the findings can shed light on the factors facilitating sustainability and spread from participants' perspectives, and participants commented on the degree to which sustainability and spread had been achieved at their sites, the findings do not provide objective evidence of the degree of sustainability and spread of the initiative at the sites.

situation awareness is a shared understanding by staff of the clinical status of patients in real time, so that any possible deterioration can be anticipated. ${ }^{1}$ The huddle is a frequent, brief meeting of staff of varying disciplines and seniority, to share information about patients, including risk of deterioration. ${ }^{1-3}$ The intention is for shared understanding of risk to lead to the mitigation of the risk and result in an increase in the team's safety culture. ${ }^{4}$ The aim of our study was to examine the challenges/barriers and facilitators to the sustainability and spread of the huddle as identified by paediatric hospital staff on four wards across England.

\section{DEFINITION OF KEY CONCEPTS}

Improvement initiatives in healthcare are often not sustained in the longer term, with 
a lack of consensus on the factors that improve sustainability. ${ }^{5-7}$ The sustainability of healthcare interventions tends to be determined by the extent to which they become integrated into daily practice, or become 'business as usual'. ${ }^{8}$ Moore $e t$ al propose that to be sustained, an intervention must continue to be supported, allow for adaptation, have changed behaviours of staff in the process, and have demonstrated clear benefits for both patients and staff. ${ }^{9}$ The spread of an innovation within and across clinical teams is often linked to sustainability. ${ }^{10}$ It has been postulated that spread within teams is a horizontal diffusion of practice, as opposed to scale-up, which is a more vertical top-down process. ${ }^{11}$

\section{METHOD}

\section{Setting for the study}

The impact of SAFE on patient safety, patient and parent experience of care, and staff working environments, was assessed through an evaluation framework. ${ }^{12}$ To qualitatively evaluate SAFE, semistructured interviews were conducted with staff at four of the 12 National Health Service (NHS) sites that took part in the first wave of implementation. The sites, two located in the north and two located in the south of England, consisted of four wards (each with 15-25 beds) across two district general hospitals and two specialist children's hospitals. Three were paediatric inpatient wards with a range of specialties (one contained a high-dependency unit; HDU), and the fourth ward was an HDU.

Qualitative data collection took place at the start of implementation (time 1; 4 months after the start of SAFE), mid-implementation (time 2; 6 months later) and during late implementation (time $3 ; 6$ months after time 2). Findings from the time 1 interviews with staff on the perceived benefits/challenges of the huddle and barriers/facilitators to its implementation during the early delivery phase have been published elsewhere. ${ }^{13}$ The focus of our study is on the time 3 (late implementation) interviews with staff to explore their perceptions of the degree of sustainability and spread of the huddle, and the challenges/barriers and facilitators to its sustainability and spread.

\section{Participants}

Twenty-three interviews were conducted with staff at late implementation (time 3). Four to seven interviews were conducted at each site. All participants had previously been interviewed at either time 1 or time 2 , or at both time-points. The final time 3 sample consisted of a range of staff members at each site: consultants $(\mathrm{N}=3)$, registrars or junior doctors $(\mathrm{N}=1)$, sisters $(\mathrm{N}=4)$, nurses $(\mathrm{N}=6)$, administration or housekeeping staff $(\mathrm{N}=1)$, ward managers and matrons $(\mathrm{N}=2)$ and specialisms such as physiotherapists, play therapists and school staff $(\mathrm{N}=6)$. See Stapley et al for further information about participant recruitment. ${ }^{13}$

\section{PATIENT AND PUBLIC INVOLVEMENT}

The SAFE programme had a parent on the steering committee who was involved in the planning of the intervention and the evaluation. It was also recommended to each clinical team that a parent and child representative were involved in intervention roll-out at a local level. Patients and families were not involved in the study reported on in this paper.

\section{Data collection}

The time 3 interviews were either conducted face to face at the sites or over the telephone by the evaluation team, using a semistructured interview schedule. Participants were asked about their perceptions of the consistency and degree of sustainability of the huddle on their ward, the spread of the huddle to other wards or to other hospital sites, and the challenges/barriers and facilitators to this. All interviews (ranging from 7 to $41 \mathrm{~min}$ in length) were audiorecorded and transcribed verbatim. As the interviews were predominantly conducted in situ, the interview lengths and depth varied according to how much time each staff member was able to take out of their working hours to speak with the evaluation team. This also meant that focus groups were not feasible, as multiple staff could not leave their duties on the ward at the same time.

\section{Data analysis}

A deductive thematic analysis was conducted to answer the following research question: What barriers/challenges and facilitators do ward staff members describe in relation to the sustaining and spread of the huddle? The content of the interview transcripts was initially coded (categorised) in a Microsoft Excel spreadsheet by the second author (DG) into two overarching categories defined by the research question: 'Barriers/Challenges'; 'Facilitators'. DG then coded the transcript extracts within these overarching categories into additional subcategories: 'Innovation', 'Context', 'Leadership', and 'Process', based on the Fleiszer $e t a l$ 's framework for factors influencing sustainability, which was developed from a comprehensive review of the literature relating to the sustainability of healthcare innovations (see table 1$).{ }^{14} 15$ We used this framework to structure our analysis of the staff interviews and consider the interplay of the four

\begin{tabular}{|c|c|c|}
\hline Factor & Facilitators & Barriers/challenges \\
\hline Innovation & Relevance and value & \\
\hline Context & Partnership with others & $\begin{array}{l}\text { Funding } \\
\text { Lack of ownership } \\
\text { or interdisciplinary } \\
\text { spread }\end{array}$ \\
\hline Leadership & $\begin{array}{l}\text { Positive senior } \\
\text { leadership commitment }\end{array}$ & $\begin{array}{l}\text { No leadership } \\
\text { commitment }\end{array}$ \\
\hline Process & $\begin{array}{l}\text { Improvement } \\
\text { methodology of test, } \\
\text { learn and adapt }\end{array}$ & \\
\hline
\end{tabular}


factors identified by Fleiszer $e t$ al within the context of SAFE. The third author (JE-C) then recoded 20\% (N=5) of the transcripts, which facilitated DG in reflecting on and refining his coding of the dataset where necessary.

\section{RESULTS}

\section{Perceived extent of sustainability and spread}

Participants across all four sites described how the huddle had become more embedded on their wards over time. All participants were confident that the huddle would continue after the end of the formal SAFE programme. Participants described the huddle as being part of their ward routine or team culture, using language like everyone 'knows the drill', or the huddle has become 'second nature'. Most participants indicated that the huddle took place when key members of staff, such as the nurse in charge, were absent. However, morning and weekday huddles had been more effectively sustained than those in the evening or at weekends.

Participants across all four the sites commented that the implementation of the huddle had become more timeefficient and smooth over time. Yet, some participants also indicated that the huddle had become 'normalised' with limitations. For instance, junior nursing staff members at one site had been consistently unable to attend the huddle over the course of the programme, as it took place away from patient bedsides. By contrast, participants at another site, which had many medical specialty teams feeding into the ward, observed that huddles mainly involving the nursing staff team had been better embedded than those also including the multidisciplinary medical team, due to their competing priorities.

At three of the sites, participants described how the spreading of the huddle to other wards had already been achieved (including contextual adaptation as necessary), and at the fourth site spreading was in process. The types of ward that the huddle had spread to included intensive care, neonatal wards, and surgical units.

\section{Innovation-related factors}

The factors related to innovation are reported in table 2. Perception of the benefits of the huddle at an organisational, team or personal level was a commonly reported facilitator to integrating it into practice. Similarly, the perceived relevance of the huddle in addressing a specific problem, such as improvement in communication between staff, was highlighted as facilitating the sustaining of the huddle and its spread to other wards.

Participants agreed that having the commitment of the whole team and educating staff on the specific benefits of the huddle, such as through the use of real-life examples (rather than academic papers), was the first step to spreading the huddle within clinical teams and then to other teams. Lack of commitment by senior staff, such as consultants, was seen as a barrier to integrating the huddle into ward practices. However, participants also commented that staff need to first understand how the huddle fits into the current procedures of the ward before being able to appreciate its potential benefits.

Participants described the importance of adapting implementation to complement existing ward practices, such as finding the right time and location for the huddle. Consistency in the time and format of the huddle was ultimately thought to be important, as was ease of implementation, brevity and ensuring that the huddle did not overlap in purpose with existing meetings on the ward, for instance 'that it does not become the same as handover', as this could diminish its utility.

Table 2 Innovation-related staff-reported challenges/barriers and facilitators to the sustainability and spread of the huddle

\begin{tabular}{|c|c|}
\hline Factor & Example quotes from staff interviews \\
\hline $\begin{array}{l}\text { Perceived relevance of the } \\
\text { huddle in addressing a need } \\
\text { or problem }\end{array}$ & $\begin{array}{l}\text { 'I think a lot of it has been through frustration with poor communication. I say frustration, } \\
\text { because sometimes it can be, you're trying to get hold of people or you think someone knows } \\
\text { something and they don't know it, so I think it's definitely improved communication' }\end{array}$ \\
\hline $\begin{array}{l}\text { Commitment of stakeholders } \\
\text { to the huddle }\end{array}$ & 'I think the main thing is that both doctors and nurses are all on board with it' \\
\hline $\begin{array}{l}\text { Fit with existing organisational } \\
\text { procedures }\end{array}$ & $\begin{array}{l}\text { 'The consultant gets tied up helping in the assessment unit. It also coincides with medicine } \\
\text { round for the nurses and it just didn't really work or take off' }\end{array}$ \\
\hline $\begin{array}{l}\text { Adaptability of the huddle to } \\
\text { the context }\end{array}$ & $\begin{array}{l}\text { 'Adapt it as time goes on, like if you think some questions [in the huddle script] are irrelevant to } \\
\text { your ward, change it and add something [that] you think is more relevant' }\end{array}$ \\
\hline $\begin{array}{l}\text { Ensuring minimal overlap with } \\
\text { existing meetings }\end{array}$ & 'It [should] not become the same as handover' \\
\hline $\begin{array}{l}\text { Demonstrating the benefits of } \\
\text { the huddle }\end{array}$ & $\begin{array}{l}\text { 'If it's sold in the right manner then you think, "I think we should do that-that would be really } \\
\text { good" }\end{array}$ \\
\hline
\end{tabular}


Table 3 Leadership-related staff-reported challenges/ barriers and facilitators to the sustainability and spread of the huddle

\begin{tabular}{ll}
\hline Factor & Example quotes from staff interviews \\
\hline $\begin{array}{l}\text { Presence and } \\
\text { influence of } \\
\text { programme } \\
\text { champion(s) }\end{array}$ & $\begin{array}{l}\text { 'Key people set it up and I think it was } \\
\text { them championing the 'come on let's } \\
\text { get together, let's start this' and just } \\
\text { persisting with it really until it becomes } \\
\text { a habit' }\end{array}$ \\
$\begin{array}{l}\text { Involvement of } \\
\text { leadership and } \\
\text { management }\end{array}$ & $\begin{array}{l}\text { 'I think perhaps next time, if we were } \\
\text { evould definitely want to involve matron, } \\
\text { or one of the ward managers who would } \\
\text { drive it' }\end{array}$ \\
\hline
\end{tabular}

\section{Leadership-related factors}

Stable, encouraging and supportive leadership from senior nursing and medical staff was considered crucial for sustaining the huddle, as shown in table 3. A clear understanding by executive and clinical leaders on other wards of the benefits of the huddle before implementation was also seen as necessary to facilitate its spread. A suggestion from participants was to have at least one 'champion' from different specialties on the ward, who would be a 'driving force' for the huddle, encourage the commitment of staff to sustaining the process, disseminate the huddle procedure to other staff, and facilitate its spread within the team and then to other wards.

\section{Process-related factors}

A key process-related facilitator to sustainability was the sharing of information with staff about the huddle. For example, one participant suggested that the huddle could be discussed at staff forums or learning events, and in induction sessions for new staff. Participants also felt that new staff could benefit from having formal training in the huddle methodology, without which the ongoing integration of the huddle into ward practices could be undermined, as new staff may not be sufficiently aware of the purpose and process of huddles. In addition, training was viewed as important in spread initiatives, alongside collaborative implementation. Process-related factors are reported in table 4 .

\section{Context-related factors}

The importance of context as a factor is reflected in table 5 . Contextual factors were more commonly reported as barriers by participants to the sustainability of the huddle. High staff turnover, large multidisciplinary medical teams, frequent changes of ward management, and a lack of senior leadership during the weekend, represented challenges to the sustaining of the huddle at the sites.

On the other hand, contextual factors were referenced by participants as facilitators in the context of the spread of the huddle. For instance, participants described how senior management staff (eg, consultants and ward managers) could use their authority to initiate the huddle innovation 'top-down', whereas front-line staff (eg, nurses) could make sure that the huddle actually happens 'bottom-up'. Thus, an effective programme team needs to comprise staff representing both groups.

In addition, participants noted that having the right level of expertise within the team is necessary to facilitate its spread to a new ward, including ideally having a staff member on that ward with experience of the huddle and knowledge of the organisation who can lead or advise on implementation. Having a culture open to innovation also facilitates the spread of the huddle. On the contrary, participants commented that dislike of or reluctance to change may prevent the huddle from being spread.

\section{DISCUSSION}

The aim of our study was to examine the challenges/barriers and facilitators to the sustainability and spread of the huddle, a quality improvement initiative and core component of the SAFE programme, as identified by paediatric hospital staff in qualitative interviews at four NHS sites across England. We used the framework proposed by Fleiszer et al to examine sustainability as influenced by innovation, context, leadership and process related factors. ${ }^{1415}$ Our study considered the interplay of these four factors in the context of SAFE, as indicated in figure 1.

\section{Innovation}

Innovation-related factors reported by participants as barriers/challenges and facilitators to sustaining the huddle included the fit of the huddle with the current

\begin{tabular}{|c|c|}
\hline Factor & Example quotes from staff interviews \\
\hline Collaboration & $\begin{array}{l}\text { 'Maybe it's the way you put it to nurses, rather than imposing it on them, people, nurses [like] } \\
\text { myself, respond better to 'this is helping a patient', rather than people saying 'you have to do this" }\end{array}$ \\
\hline $\begin{array}{l}\text { Planning and education } \\
\text { around implementation }\end{array}$ & 'Training in how to do a huddle. Yes, that would be useful' \\
\hline
\end{tabular}

Communication about the 'The nurse in charge [should] try and get everyone together to explain what's going to happen' huddle

Use of performance monitoring systems
'If you wanted to improve your quality, look at your data. [Then] say to the team 'these are our issues - what should we work on?' because [for] some things you just need to do a proper PDSA(Plan, Do, Study, Act)cycle' 
Table 5 Context-related staff-reported challenges/barriers and facilitators to the sustainability and spread of the huddle

\begin{tabular}{|c|c|}
\hline Factor & Example quotes from staff interviews \\
\hline Management structure & $\begin{array}{l}\text { 'There was another change and another change [in ward manager] and that is not conducive to } \\
\text { supporting a ward to implement a new quality [improvement] initiative' }\end{array}$ \\
\hline Organisational culture & $\begin{array}{l}\text { 'I think we were relatively lucky that there was that kind of culture to some extent. So, getting } \\
\text { other people on board was maybe easier' }\end{array}$ \\
\hline Staffing structure & $\begin{array}{l}\text { 'I think it's still hit and miss because we haven't extended the consultant cover to cover } \\
\text { weekends' }\end{array}$ \\
\hline
\end{tabular}

processes and procedures of the ward, adapting the huddle as necessary to reflect the context, perceptions of the benefits and added value of the huddle, having the commitment of the whole team, consistency and brevity of the huddle, and perceptions of the relevance of the huddle in addressing a specific need.

The results are consistent with existing reviews of the factors influencing the spread of an innovation, in which the perceived relevance and benefits to the adopters' role and local context are essential to embed the innovation into day-to-day practice. ${ }^{16}{ }^{17}$ Compatibility with and adaptation to the local context are also crucial to facilitate sustainability and successful spread of the intervention. ${ }^{16} 17$ Indeed, within SAFE, teams were encouraged to

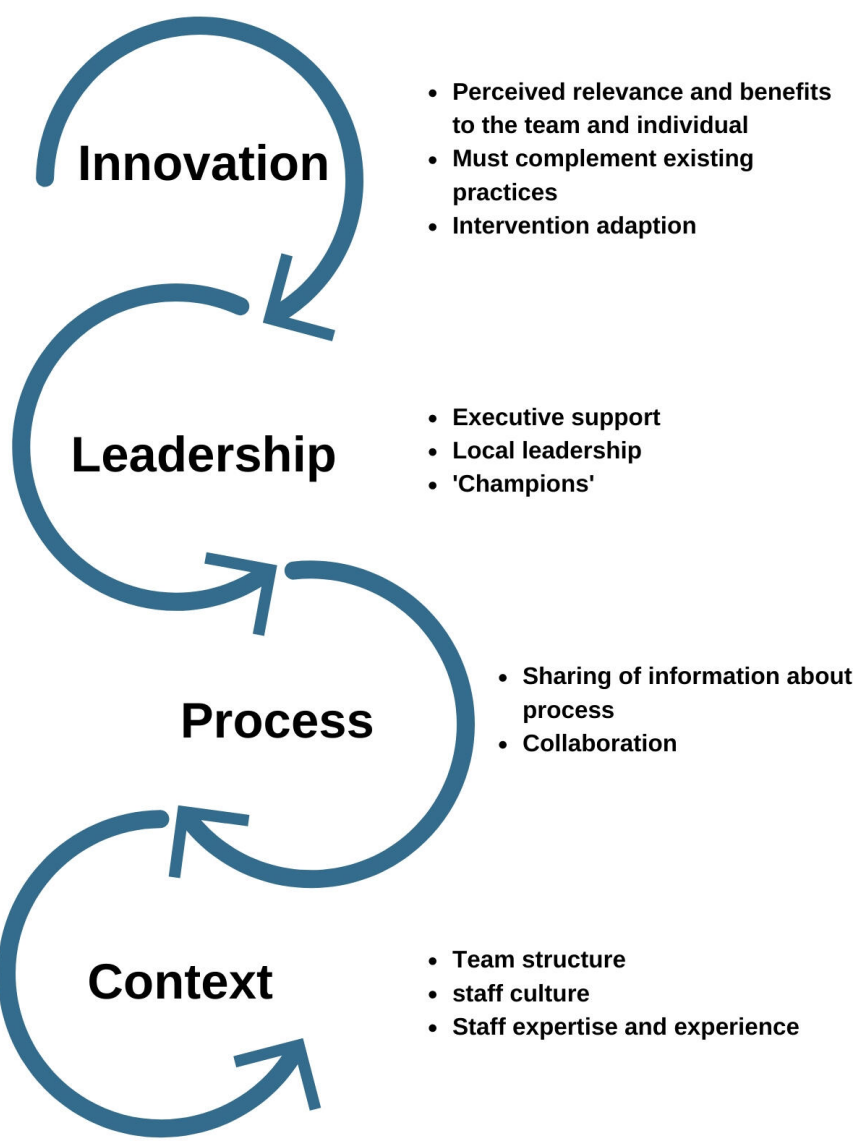

Figure 1 Construct for spread and sustainability. implement the intervention to fit with their own context and environment, using a human factors approach, ${ }^{18}$ and ensuring the relative advantage of the new intervention over the previous ward process. ${ }^{17}$

\section{Leadership}

Participants highlighted the importance of senior nursing and medical leadership, as well as having champions of the intervention within the organisation, as key facilitators to ensuring integration into ward practices and spread to other wards. The implementation of the huddle in SAFE was based on the clinical microsystem theory, in which local clinical leadership is a fundamental part of change. ${ }^{19}$ Previous research suggests that senior leadership is needed to provide quality assurance and support or drive for the intervention from the start. ${ }^{20}$

\section{Process}

Participants highlighted the importance of collaborative implementation and shared learning in various formats to facilitate the sustaining and spread of the huddle. The provision of training and education for new staff about the huddle was also seen as necessary to facilitate its ongoing sustainability and spread. This supports previous research, which suggests that implementation should be conducted collaboratively in a culture open to innovation and sustainability to be able to learn, generalise and scale-up. $^{21}$

\section{Context}

Implementation research has emphasised the importance of studying the context in which the improvement programme is to be implemented, to understand why implementation succeeds or fails. ${ }^{22-24}$ Moreover, the learning approach to the huddle was developed in accordance with the Dynamic Sustainability Framework proposed by Chambers et al, which emphasises the importance of context and the fit of the intervention to the different and changing context. ${ }^{25}$ Participants indicated that factors, such as high staff turnover and a large multidisciplinary medical team with competing priorities, were barriers to the sustainability of the huddle. Yet, having a ward culture open to innovation could facilitate its spread. In line with this, Horton et al have reported on the 
factors required for the successful adoption of an innovation, including the importance of the local environment being receptive to any changes required. ${ }^{17}$

\section{Limitations}

It is possible that staff with more negative views of SAFE may have felt less comfortable about volunteering to be interviewed, leading to an over-representation of staff with more positive views. While the four sites in our study were selected to be representative of paediatric settings in England and to ensure good geographical spread, the transferability of our findings to other contexts may be limited. We do not know, for example, whether the sites in our sample had a more or less supportive culture than others. It is also beyond the scope of this study to be able to comment objectively on the success and actual degree of the sustainability and spread of the huddle.

\section{CONCLUSIONS AND IMPLICATIONS}

Our findings reflect earlier results from the initial phase of SAFE that successful implementation of a new safety improvement initiative requires senior medical and nursing staff leadership or 'championing', a fit with existing ward practices, and staff perceptions of the benefits and understanding of the purpose of the initiative. ${ }^{13}$ Moreover, some of the barriers identified during early implementation were similarly identified during late implementation, such as reluctance to change or staff members being unable to attend the huddle due to its location on the ward or their competing responsibilities. ${ }^{13}$ This suggests that in planning an intervention, one needs to take these factors into account, and adapt practice as necessary, to ensure both successful initial implementation and then later sustainability.

In addition, the context may change over time, so planning for sustainability requires constant studying of the context to ensure that potential risks to sustainability are addressed. ${ }^{23}$ The attention to ongoing benefit of the intervention in different contexts is essential. ${ }^{25}$ The study period was for 2 years and concentrated on four hospitals. Although we cannot report on the longerterm sustainment of the intervention at the sites, we have identified the factors that appear to be important to enhance the potential for sustainability, which other clinical teams, including those within future waves of the SAFE programme, can apply to facilitate and improve the sustainability and spread of healthcare innovations in their settings.

\section{Twitter Peter Lachman @PeterLachman}

Acknowledgements The authors would like to thank the staff members at the four hospitals who generously shared their experiences and views with us. We also thank the other members of the evaluation team for their contributions to this study-Evelyn Sharples, Dr Jacqueline Hayes and Amy Ramsay. Megan Peng, Project Manager at RCPCH, contributed to an early draft of the paper. The project implementation was led by the Royal College of Paediatrics and Child Health (RCPCH) and supported by the UCL Partners Academic Health Science Network.

Contributors All of the authors were involved in the conceptualisation of the research. ES and DG (researchers in the evaluation team) led the data collection.
DG led the data analysis under the supervision of ES. J E-C contributed to analyses of the data. JE-C and JD (lead researchers in the evaluation team) contributed to the research design. PL (clinical lead for the SAFE programme) conducted the literature review and led on the drafting of the introduction and discussion sections of the paper. DG and ES contributed to the overall drafting of this paper and led on the writing of the method and results sections. All authors approved the final version of the paper.

Funding Situation Awareness For Everyone (S.A.F.E) is a Health Foundation funded programme; both the implementation of S.A.F.E and the evaluation were funded by the Health Foundation. This work was also supported by funding from WellChild; the funding was specifically to support evaluation work around the perspectives of parents and young people and to support patient and parent involvement in the research. This programme of work and evaluation was also supported by the Royal College of Paediatrics and Child Health (RCPCH), which leads on the delivery of the programme. JD was also partly supported by the National Institute for Health Research (NIHR) Collaboration for Leaderships in Applied Health Research and Care (CLAHRC) North Thames at Bart's Health NHS Trust.

Disclaimer The views expressed are those of the authors and not necessarily those of the NHS, the NIHR, the Department of Health, or RCPCH.

Competing interests PL led the implementation of the SAFE programme and was not involved in the data collection and analysis. ES, as senior author, oversaw the data collection and data analysis and approved the final version of the paper.

Patient and public involvement Patients and/or the public were involved in the design, or conduct, or reporting, or dissemination plans of this research. Refer to the Methods section for further details.

Patient consent for publication Not required.

Ethics approval Ethical approval was granted by the Dulwich Research Ethics Committee (REC reference: 14/L0/0875).

Provenance and peer review Not commissioned; externally peer reviewed.

Data availability statement Data are available on reasonable request. This is part of a larger study and four papers have already been published. This paper reports on a specific analysis and does not compete with other data held. All data are held by the research team at the Anna Freud Centre. Additional summary data are available on request to the authors.

Open access This is an open access article distributed in accordance with the Creative Commons Attribution Non Commercial (CC BY-NC 4.0) license, which permits others to distribute, remix, adapt, build upon this work non-commercially, and license their derivative works on different terms, provided the original work is properly cited, appropriate credit is given, any changes made indicated, and the use is non-commercial. See: http://creativecommons.org/licenses/by-nc/4.0/.

\section{ORCID iD}

Peter Lachman http://orcid.org/0000-0001-5120-5776

\section{REFERENCES}

1 Brady PW, Muething S, Kotagal U, et al. Improving situation awareness to reduce unrecognized clinical deterioration and serious safety events. Pediatrics 2013;131:e298-308.

2 Goldenhar LM, Brady PW, Sutcliffe KM, et al. Huddling for high reliability and situation awareness. BMJ Qual Saf 2013;22:899-906.

3 Provost SM, Lanham HJ, Leykum LK, et al. Health care huddles: managing complexity to achieve high reliability. Health Care Manage Rev 2015;40:2-12.

4 Taitz J. Building a culture of safety in pediatrics and child health. Curr Treat Options Pediatr 2015;1:253-61.

5 Scheirer MA. Linking sustainability research to intervention types. Am J Public Health 2013;103:e73-80.

6 Wiltsey Stirman S, Kimberly J, Cook N, et al. The sustainability of new programs and innovations: a review of the empirical literature and recommendations for future research. Implement Sci 2012;7:17.

7 Scheirer MA, Dearing JW. An agenda for research on the sustainability of public health programs. Am J Public Health 2011;101:2059-67.

8 Greenhalgh T, Voisey C, Robb N. Interpreted consultations as 'business as usual'? An analysis of organisational routines in general practices. Sociol Health IIIn 2007;29:931-54.

9 Moore JE, Mascarenhas A, Bain J, et al. Developing a comprehensive definition of sustainability. Implement Sci 2017;12:110. 
10 Jeffcott $\mathrm{S}$. The spread and sustainability of quality improvement in healthcare. NHS Scotland, 2014. Available: https://qi.elft.nhs.uk/wpcontent/uploads/2015/05/the-spread-and-sustainability-ofqualityimprovement-in-healthcare-pdf.pdf [Accessed 9 Feb 2021].

11 Shaw J, Tepper J, Martin D. From pilot project to system solution: innovation, spread and scale for health system leaders. BMJ Leader 2018;2:87-90.

12 Deighton J, Edbrooke-Childs J, Stapley E, et al. Realistic evaluation of situation awareness for everyone (safe) on paediatric wards: study protocol. BMJ Open 2016;6:e014014.

13 Stapley E, Sharples E, Lachman P, et al. Factors to consider in the introduction of huddles on clinical wards: perceptions of staff on the safe programme. Int J Qual Health Care 2018;30:44-9.

14 Fleiszer AR, Semenic SE, Ritchie JA, et al. The sustainability of healthcare innovations: a concept analysis. J Adv Nurs 2015;71:1484-98.

15 Fleiszer AR, Semenic SE, Ritchie JA, et al. An organizational perspective on the long-term sustainability of a nursing best practice guidelines program: a case study. BMC Health Serv Res 2015;15:535-41.

16 Rodgers EM. Diffusion of innovations. 5th edn. New York: Simon \& Schulster, 2003.

17 Horton T, Illingworth J, Warburton W. The spread challenge: how to support the successful uptake of innovations and improvements in health care. Health Foundation, 2018. Available: https://www. health.org.uk/publications/the-spread-challenge [Accessed 9 Feb 2021].

18 Carayon P, Schoofs Hundt A, Karsh B-T, et al. Work system design for patient safety: the SEIPS model. Qual Saf Health Care 2006;15(Suppl 1):i50-8.

19 Nelson EC, Batalden PB, Huber TP, et al. Microsystems in health care: Part 1. learning from high-performing front-line clinical units. Jt Comm J Qual Improv 2002;28:472-93.

20 Lachman P, Nicklin W. Effectively leading for quality. Healthc Manage Forum 2017;30:233-6.

21 Greenhalgh T, Robert G, Macfarlane F, et al. Diffusion of innovations in service organizations: systematic review and recommendations. Milbank Q 2004;82:581-629.

22 Dixon-Woods M, ed. Perspectives on context: A selection of essays considering the role of context in successful quality improvement. The Health Foundation, 2014. https://www.health.org.uk/sites/ default/files/PerspectivesOnContext_fullversion.pdf

23 Hawe P. Lessons from complex interventions to improve health. Annu Rev Public Health 2015;36:307-23.

24 Øvretveit J, Dolan-Branton L, Marx M, et al. Adapting improvements to context: when, why and how? Int J Qual Health Care 2018;30:20-3.

25 Chambers DA, Glasgow RE, Stange KC. The dynamic sustainability framework: addressing the paradox of sustainment amid ongoing change. Implement Sci 2013;8:117-27. 